\title{
Data Descriptor \\ Impact of p53 Knockout on Protein Data Set of HaCaT Cells in Confluent and Subconfluent Conditions
}

\author{
Alexander L. Rusanov *, Daniil D. Romashin, Peter M. Kozhin, Maxim N. Karagyaur, Dmitry S. Loginov (D), \\ Olga V. Tikhonova, Victor G. Zgoda and Nataliya G. Luzgina
}

check for updates

Citation: Rusanov, A.L.; Romashin, D.D.; Kozhin, P.M.; Karagyaur, M.N.; Loginov, D.S.; Tikhonova, O.V.; Zgoda, V.G.; Luzgina, N.G. Impact of p53 Knockout on Protein Data Set of HaCaT Cells in Confluent and Subconfluent Conditions. Data 2022, 7, 27. https://doi.org/10.3390/ data7030027

Academic Editor: Juraj Gregáň

Received: 30 December 2021

Accepted: 17 February 2022

Published: 23 February 2022

Publisher's Note: MDPI stays neutral with regard to jurisdictional claims in published maps and institutional affiliations.

Copyright: (C) 2022 by the authors. Licensee MDPI, Basel, Switzerland. This article is an open access article distributed under the terms and conditions of the Creative Commons Attribution (CC BY) license (https:// creativecommons.org/licenses/by/ $4.0 /)$.
V. N. Orekhovich Research Institute of Biomedical Chemistry, 119121 Moscow, Russia; dromashin@ibmc.msk.ru (D.D.R.); p.kozhin@g.nsu.ru (P.M.K.); mkaragyaur@ibmc.msk.ru (M.N.K.); dmitry.loginov@biomed.cas.cz (D.S.L.); ovt@ibmh.msk.su (O.V.T.); vic@ibmh.msk.su (V.G.Z.); ngluzgina@ibmc.msk.ru (N.G.L.)

* Correspondence: rusanov@ibmc.msk.ru

\begin{abstract}
The immortalized keratinocytes, $\mathrm{HaCaT}$, are a popular model for skin research (toxicity, irritation, allergic reactions, or interaction of cells). They maintain a stable keratinocyte phenotype and respond to keratinocyte differentiation stimuli. However, programs of stratification and expression of differentiation markers in $\mathrm{HaCaT}$ keratinocytes are aberrant. In $\mathrm{HaCaT}$ cells, there are two mutant p53 alleles (i.e., R282Q and H179Y) that contain gain-of-function (GOF) mutations resulting from spontaneous immortalization (mutp53). At the same time, mutp53 acts as a transcription factor and also affects the interaction of p63 protein with its transcription targets. Proteins of the p53 family are crucial for regulation of proliferation and differentiation processes in human keratinocytes, although the involvement of mutp53 in these processes is not fully clear. We present data sets obtained as a result of high-performance proteomic analysis of immortalized HaCaT keratinocytes with p53 knockout in two different states, subconfluent and confluent, which are characterized by different intensites of cell differentiation processes. To obtain the proteomic profiles of the cells, we applied LC-MS/MS measurements processed with MaxQuant software (version 1.6.3.4).
\end{abstract}

Keywords: keratinocyte; p53; proteome; LC-MS/MS; knockout; confluent

\section{Introduction}

Proteins of the p53 family take an active part in the regulation of processes of proliferation and differentiation of human keratinocytes both in normal physiology and keratinization disorders [1,2]. It is noteworthy that some of p53-dependent genes, such as P21 and 14-3-3 $\sigma$, are activated during keratinocyte differentiation [3]. In some studies, it has been demonstrated that psoriasis and atopic dermatitis, with aberrant differentiation of keratinocytes, are accompanied by an increase in p53 gene expression $[4,5]$.

Another transcription factor of the p53 family is the p63 protein, coded by TP63, the master gene of keratinocyte differentiation. It plays a key role in epidermal development, where it is directly involved in the regulation of numerous target genes crucial for the implementation of the processes of keratinocyte proliferation, differentiation, and adhesion $[1,6]$. The features of the functioning of these proteins in the cells of the immortalized $\mathrm{HaCaT}$ keratinocyte cell line have been studied to a lesser extent. In HaCaT cells, there are two mutant TP53 alleles (i.e., R282Q and H179Y) that contain gain-of-function (GOF) mutations [7]. At least two mechanisms of the GOF of mutant p53 are known: it affects the functions of $\mathrm{p} 63 / \mathrm{p} 73$ by inhibiting their binding to DNA [8], or it binds to new DNA sites by interacting with other transcription factors (NF-Y, E2F1, NF-KB, VDR, and p63) $[9,10]$. More than seven thousand DNA binding sites are known for Mutp53 in HaCaT cells [11]. In addition, in contrast to normal human keratinocytes, HaCaT cells mainly express the $\Delta \mathrm{N}$ p63 isoform (synthesized from an internal promoter and lacks the $N$-terminal transactivation domain), and the TA isoform (with $N$-terminal transactivation domain) is practically 
not detected [12]. Since the HaCaT cell line is the most popular model of normal human keratinocytes, studying the functioning of p53 family proteins in these cells is important for understanding the limitations of this model system.

This study aimed to obtain data on the proteomic profile of HaCaT cells with knockedout TP53 and WT HaCaT in two different states: subconfluent and confluent. It is known that when the confluent state is reached, the processes of differentiation of human keratinocytes turn out to be the most intense [13].

\section{Data Description}

The data set contained "RAW" files generated by high-throughput proteomic analysis of wild-type HaCaT keratinocytes (WT HaCaT) and p53 knockout HaCaT keratinocytes (dp53 HaCaT). Sample information is presented in Table 1. The data set included 16 biological samples. Data files are listed in Table 2.

Table 1. Information about the studied cell samples and the number of repeats.

\begin{tabular}{ccccc}
\hline Cell Type & $\begin{array}{c}\text { WT HaCaT } \\
\text { Subconfluent }\end{array}$ & $\begin{array}{c}\text { dp53 HaCaT } \\
\text { Subconfluent }\end{array}$ & $\begin{array}{c}\text { WT HaCaT } \\
\text { (100\% Confluence, 72 h) }\end{array}$ & $\begin{array}{c}\text { dp53 HaCaT } \\
\text { (100\% Confluence, 72 h) }\end{array}$ \\
\hline Number of samples & 4 & 4 & 4 & 4 \\
\hline Technical repeats per sample & $3-5$ & 3 & 3 & 3 \\
\hline
\end{tabular}

Western blot was employed for knockout verification (Figure 1).

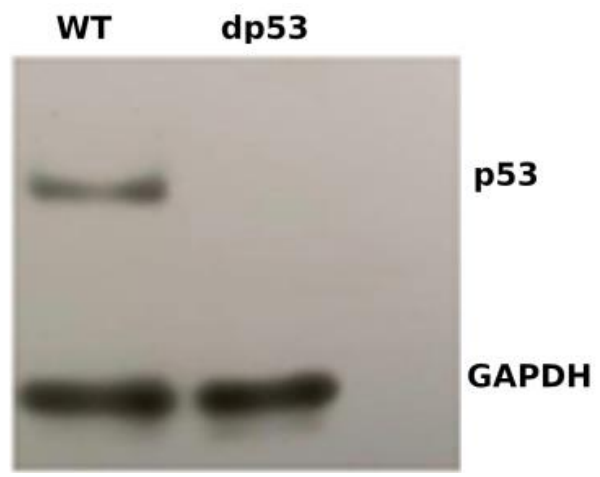

Figure 1. p53 expression in HaCaT cells by Western blot. WT—wild type HaCaT; dp53-p53 knockout HaCaT.

Comparison of confluent wild-type and dp53 mutant protein profiles resulted in the identification of 405 proteins. Out of them, 224 were identified in both samples and 181 were found either in WT or mutant. Meanwhile, the overall protein number for the nonconfluent pair (i.e., WT and dp53 mutant) was 803, including 398 common and 405 unique proteins. Using $t$-tests, statistically significant differences in the production level were found for nine and three proteins for confluent and nonconfluent cells, respectively. The common trend for both pairs was a significantly lower number of unique proteins (detected only in one condition) for the WT (9 vs. 153 and 32 vs. 373 for the confluent and nonconfluent samples, respectively).

In addition, the protein profiles of the confluent samples were compared with the respective nonconfluent ones. The common trend was a decreased number of produced proteins in the nonconfluent samples. Overall, 788 and 785 proteins were identified for WT and mutant samples, respectively. Out of them, 536 and 488 proteins were only found in confluent WT and dp53 mutant, respectively; whereas only 4 and 14 proteins were characteristic for the respective nonconfluent analogs. In addition, for the confluent/nonconfluent mutant pairs, 106 proteins with statistically significant expression were determined (Figure 2). 
Table 2. Data set description.

\begin{tabular}{|c|c|c|c|}
\hline Sample & File Name & Type & Size $(\mathbf{M b})$ \\
\hline WT 72 h s1_TR1 & 110821CKP_LNRA01.raw & RAW & 792 \\
\hline WT 72 h s1_TR2 & 110821CKP_LNRA01_210811225153.raw & RAW & 702 \\
\hline WT 72 h s1_TR3 & 110821CKP_LNRA01_210812002917.raw & RAW & 771 \\
\hline WT 72 h s2_TR1 & 110821CKP_LNRA02.raw & RAW & 890 \\
\hline WT 72 h s2_TR2 & 110821CKP_LNRA02_210812045125.raw & RAW & 812 \\
\hline WT 72 h s2_TR3 & 110821CKP_LNRA02_210812062849.raw & RAW & 787 \\
\hline WT 72 h s3_TR1 & 110821CKP_LNRA03.raw & RAW & 657 \\
\hline WT 72 h s3_TR2 & 110821CKP_LNRA03_210812105056.raw & RAW & 700 \\
\hline WT 72 h s3_TR3 & 110821CKP_LNRA03_210812122818.raw & RAW & 690 \\
\hline WT 72 h s4_TR1 & 110821CKP_LNRA04.raw & RAW & 710 \\
\hline WT 72 h s4_TR2 & 110821CKP_LNRA04_210812165027.raw & RAW & 679 \\
\hline WT 72 h s4_TR3 & 110821CKP_LNRA04_210812182749.raw & RAW & 707 \\
\hline dP53 72 h s1_TR1 & 110821CKP_LNRA05.raw & RAW & 763 \\
\hline dP53 72 h s1_TR2 & 110821CKP_LNRA05_210812224958.raw & RAW & 711 \\
\hline dP53 72 h s1_TR3 & 110821CKP_LNRA05_210813002725.raw & RAW & 724 \\
\hline dP53 72 h s2_TR1 & 110821CKP_LNRA06.raw & RAW & 737 \\
\hline dP53 72 h s2_TR2 & 110821CKP_LNRA0̄06_210813044935.raw & RAW & 678 \\
\hline dP53 72 h s2_TR3 & 110821CKP_LNRA06_210813062700.raw & RAW & 676 \\
\hline dP53 72 h s3_TR1 & 110821CKP_LNRA07.raw & RAW & 670 \\
\hline dP53 72 h s3_TR2 & 110821CKP_LNRA07_210813104909.raw & RAW & 684 \\
\hline dP53 72 h s3_TR3 & 110821CKP_LNRA07_210813122634.raw & RAW & 664 \\
\hline dP53 72 h s4_TR1 & 110821CKP_LN̄EA08.raw & RAW & 711 \\
\hline dP53 72 h s4_TR2 & 110821CKP_LNRA0̄8_210813164846.raw & RAW & 757 \\
\hline dP53 72 h s4_TR3 & 110821CKP_LNRA08_210813182610.raw & RAW & 715 \\
\hline WT subconf. s1_TR1 & 110821CKP_LNRA09.raw & RAW & 689 \\
\hline WT subconf. s1_TR2 & 110821CKP_LNRA09_210813224817.raw & RAW & 683 \\
\hline WT subconf. s1_TR3 & 110821CKP_LNRA09_210814002539.raw & RAW & 690 \\
\hline WT subconf. s2_TR1 & 110821CKP_LNRA10.raw & RAW & 671 \\
\hline WT subconf. s2_TR2 & 110821CKP_LNRA10_210814044742.raw & RAW & 690 \\
\hline WT subconf. s2_TR3 & 110821CKP_LNRA10_210814062506.raw & RAW & 679 \\
\hline WT subconf. s3_TR1 & 110821CKP_LNRA11.raw & RAW & 646 \\
\hline WT subconf. s3_TR2 & 110821CKP_LNRA11_210814104709.raw & RAW & 656 \\
\hline WT subconf. s3_TR3 & 110821CKP_LNRA11_210817210241.raw & RAW & 850 \\
\hline WT subconf. s3_TR4 & 110821CKP_LNRA11_210817224002.raw & RAW & 789 \\
\hline WT subconf. s3_TR5 & 110821CKP_LNRA11_210818001729.raw & RAW & 797 \\
\hline WT subconf. s4_TR1 & 110821CKP_LNRA12.raw & RAW & 799 \\
\hline WT subconf. s4_TR2 & 110821CKP_LNRA12_210818043938.raw & RAW & 771 \\
\hline WT subconf. s4_TR3 & 110821CKP_LNRA12_210818061703.raw & RAW & 755 \\
\hline dP53 subconf. s1_TR1 & 110821CKP_LNRA13.raw & RAW & 757 \\
\hline dP53 subconf. s1_TR2 & 110821CKP_LNRĀ̄13_210818103910.raw & RAW & 761 \\
\hline dP53 subconf. s1_TR3 & 110821CKP_LNRA13_210818121633.raw & RAW & 774 \\
\hline dP53 subconf. s2_TR1 & 110821CKP_LNRA14.raw & RAW & 696 \\
\hline dP53 subconf. s2_TR2 & 110821CKP_LNRA14_210818163844.raw & RAW & 1054 \\
\hline dP53 subconf. s2_TR3 & 110821CKP_LNRA14_210818181609.raw & RAW & 1043 \\
\hline dP53 subconf. s3_TR1 & 110821CKP_LNRA15.raw & RAW & 1144 \\
\hline dP53 subconf. s3_TR2 & 110821CKP_LNRA15_210818223820.raw & RAW & 1079 \\
\hline dP53 subconf. s3_TR3 & 110821CKP_LNRA15_210819001551.raw & RAW & 1077 \\
\hline dP53 subconf. s4_TR1 & 110821CKP_LNRA16.raw & RAW & 1045 \\
\hline dP53 subconf. s4_TR2 & 110821CKP_LNRĀ16_210819043801.raw & RAW & 1046 \\
\hline \multirow[t]{3}{*}{ dP53 subconf. s4_TR3 } & 110821CKP_LNRA16_210819061530.raw & RAW & 1062 \\
\hline & SEARCH.rar & SEARCH & 1011 \\
\hline & checksum.txt & OTHER & 0.0008 \\
\hline
\end{tabular}



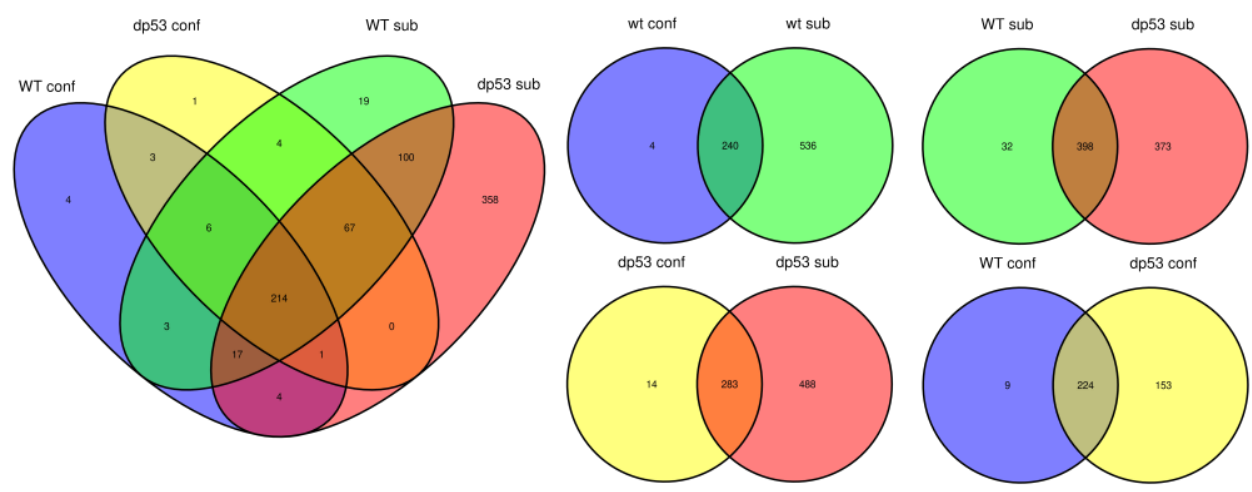

Figure 2. Venn diagram of identified proteins. The numbers of proteins detected are indicated by numbers. WT sub-wild-type HaCaT in the subconfluent state; WT conf-wild-type HaCaT in the confluent state; dp53 sub-p53 knockout HaCaT in the subconfluent state; dp53 conf-p53 knockout $\mathrm{HaCaT}$ in the confluent state.

To evaluate the mutual influence of mutation and confluency onto protein profiles of cells, two-way ANOVA analysis was performed. Overall, 511 proteins were significant as an interception of these two parameters. Most of these significant proteins were involved in different cellular metabolic processes $(32.5 \%)$ or related to cellular component organization or biogenesis (20.2\%) (Figure 3). The STRING analysis of differently expressed proteins for confluent cells is presented in Figure 4.

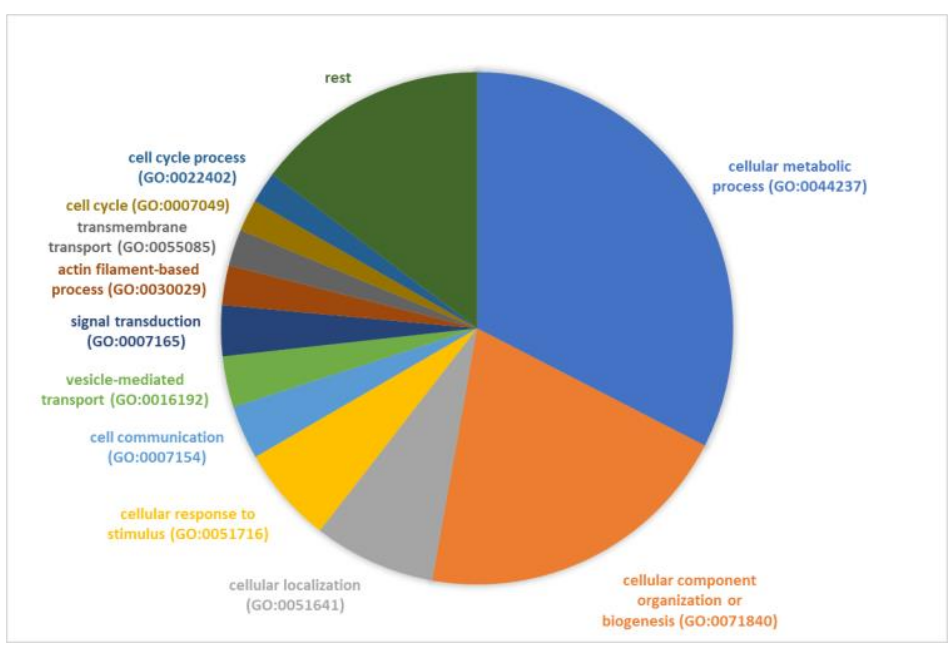

Figure 3. Gene Ontology analysis by biological function of proteins significant for the interaction effect $(p<0.05)$ determined by two-way ANOVA analysis. The samples were categorized based on the presence of mutation and confluency.

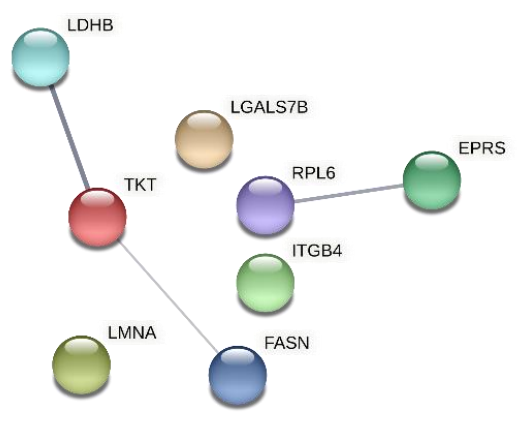

Figure 4. STRING analysis of differently expressed proteins for confluent cells. 


\subsection{Value of the Data}

- $\quad$ The data set presents wild-type and p53 knockout human immortalized HaCaT keratinocyte proteomes that can be examined to determine differences;

- $\quad$ Provided data may be valuable to researchers developing in vitro skin models;

- $\quad$ Provided data will be useful for scientists investigating epidermal stratification;

- $\quad$ "RAW" data files are provided and can be processed by researchers using preferable bioinformatics tools and compared to their data.

\subsection{Data Records}

Proteomic data were deposited to the ProteomeXchange Consortium via the PRIDE partner repository (http:/ / www.proteomexchange.org/Project, accessed on 29 December 2021, accession: PXD030700).

\section{Materials and Methods}

\subsection{Reagents}

Sodium chloride, taurocholic acid sodium salt (TCA), and acetonitrile were provided by Merck (Darmstadt, Germany). Formic acid was from ACROS Organics (Geel, Belgium), and tris-(2-carboxyethyl)-phosphine (TCEP), methanol, and trifluoroacetic acid (TFA) were from Fluka (Buchs, Switzerland). Modified trypsin was obtained from Promega (Madison, WI, USA). For cell cultivation, we used DMEM/F:12 medium (1:1), GlutaMAX, fetal bovine serum, PBS, and penicillin/streptomycin (all from Gibco, Waltham, MA, USA).

\subsection{Cells and Culture Conditions}

HaCaT cells were obtained from DKFZ, Heidelberg, and cultured in DMEM/F:12 medium (1:1, Gibco, Waltham, MA, USA) supplemented with 1\% GlutaMAX (Thermo Fisher Scientific, Waltham, MA, USA), penicillin/streptomycin $(100 \mathrm{UI} / \mathrm{mL}$ and $100 \mu \mathrm{g} / \mathrm{mL}$, Gibco, Waltham, MA, USA), and 10\% v/v fetal bovine serum (Gibco, Waltham, MA, USA). Cell cultivation was carried out in $25 \mathrm{~cm}^{2}$ tissue-treated flasks or $60 \mathrm{~mm}$ Petri dishes (Corning, Corning, NY, USA) with culture medium replaced fresh every two days. Cells were harvested by trypsinization after 3 day culture in $100 \%$ confluence for the confluent condition or after achieving $70 \%$ confluence for the subconfluent condition.

\subsection{CRISPR/Cas TP53 Downregulation}

Cas9n D10A from S. pyogenes was employed to perform TP53 gene knockout. PX461 (Addgene, \#48140) encoding Cas9n (D10A), appropriate gRNAs, and the GFP reporter gene was used for transfection. The sequences of paired gRNA are listed below:

gRNA 1-GGAAGCTCCCAGAATGCCAG; gRNA 2-GCATTGTTCAATATCGTCCG.

HaCaT cells were seeded at a concentration of $1 \times 10^{5}$ per $\mathrm{mL}$ of medium in 6 -well plate $48 \mathrm{~h}$ prior to transfection and grown under standard conditions. The cells were transfected with PEI (0.5 mg/mL, Polysciences, Inc., \#23966-1, Warrington, PA, USA) and $2 \mu \mathrm{g}$ of each vector. After 2 days, cells expressing GFP were sorted via BD FACSAria III instrument (BD Biosciences, Franklin Lakes, NJ, USA) directly to 96-well plates and grown until clonal analysis.

\subsection{Sequence Analysis}

The p53 deficient clones were grown under standard conditions for $48 \mathrm{~h}$ up to a $70 \%$ confluence. Total DNA was isolated with the ExtractDNA Blood \& Cells kit (Evrogen, Moscow, Russia, \#BC111M). The area of alleged editing was amplified with the following primers:

F-AAAGTTCTGCATCCCCAGGAG; R-GCAGTCAGATCCTAGCGTCG. 
The Sanger sequencing was performed by Evrogen, Moscow, Russia. The quantitative assessment of genome editing was performed with TIDE software as described [14].

\subsection{Western Blot}

Verification of knockout was performed using $8 \%$ sodium SDS-PAGE, and $1 \times 10^{6}$ of wild-type and p53 knockout cells were harvested via trypsinization for Western blot analysis. The cell pellet was lysed in RIPA buffer $(150 \mathrm{mM} \mathrm{NaCl}, 1 \%$ Triton X-100, 0.5\% sodium deoxycholate, $0.1 \%$ SDS, $50 \mathrm{mM}$ Tris, and $\mathrm{pH} 8.0$ ).

The proteins were transferred onto PVDF membrane following immunoblotting with anti-p53 (clone BP-53-12, PrimeBioMed, Moscow, Russia) and anti-GAPDH (Abcam, ab9485, Cambridge, UK) antibodies as loading control. Alkaline phosphatase-conjugated secondary antibodies were used for primary antibodies recognition. The chemiluminescent reaction was detected via X-ray film.

\subsection{Protein Extraction for MS Analysis}

The cell pellets were lysed in $100 \mu \mathrm{L}$ of lysis buffer (4\% SDS in PBS, pH 7.4). The lysates were incubated under rotation at room temperature for $20 \mathrm{~min}$ following $5 \mathrm{~min}$ of incubation at $95^{\circ} \mathrm{C}$. The samples were cooled down and sonicated with Bandelin 2070 (Bandelin, Germany) by three 20 s cycles at $90 \%$ power. The methanol-chloroform method was applied for protein precipitation [15]. Briefly, $400 \mu \mathrm{L}$ of methanol was added to the lysates. The mixtures were vortexed; then, $100 \mu \mathrm{L}$ of chloroform and $300 \mu \mathrm{L}$ of $\mathrm{ddH}_{2} \mathrm{O}$ were added. The mixtures were vortexed and centrifuged at $14,000 \times g$ for $2 \mathrm{~min}$. The aqueous layer was discarded and $400 \mu \mathrm{L}$ of methanol was added. The samples were mixed by vortex and centrifuged for $5 \mathrm{~min}$ at $14,000 \times \mathrm{g}$. The methanol was removed, and the pellets were speed-dried for $5 \mathrm{~min}$ at $45^{\circ} \mathrm{C}$ with the Eppendorf Concentrator 5301 (Eppendorf, Hamburg, Germany).

\subsection{Sample Preparation for MS Analysis}

Protein concentrations were determined with the BCA assay kit (Pierce ${ }^{\mathrm{TM}}$ BCA Protein Assay Kit, Waltham, MA, USA). Protein extracts (50 $\mu \mathrm{g}$ ) were diluted in $20 \mu \mathrm{L}$ of denaturation buffer (5M urea, 1\% TCA, 15\% acetonitrile, $50 \mathrm{mM}$ phosphate buffer at $\mathrm{pH} 6.3$, and $300 \mathrm{mM}$ sodium chloride). Then, $5 \mu \mathrm{L}$ of $25 \mathrm{mM}$ TCEP in $0.1 \mathrm{M}$ ammonium bicarbonate was added to the mixtures followed by $30 \mathrm{~min}$ incubation in the dark. To quench the remaining IAA, $5 \mu \mathrm{L}$ of $300 \mathrm{mM}$ DTT in $0.1 \mathrm{M}$ ammonium bicarbonate was added. The mixtures were diluted in $0.1 \mathrm{M}$ ammonium bicarbonate to a final volume of $200 \mu \mathrm{L}$. Subsequently, trypsin in enzyme: protein ratio 1:50 was added for in-solution digestion. The samples were incubated overnight at $37^{\circ} \mathrm{C}$ under rotation and centrifuged at $10{ }^{\circ} \mathrm{C}$ and $12,000 \times g$ for $10 \mathrm{~min}$. Supernatants were collected and purified with C18 ZipTip following the supplier's protocol [16]. Briefly, the columns were washed with $0.1 \%$ trifluoroacetic acid (TFA) in acetonitrile, then equilibrated twice with $0.1 \%$ TFA in $\mathrm{ddH}_{2} \mathrm{O}$. The samples were applied to the ZipTips and washed thrice with $0.1 \%$ TFA and 5\% methanol in $\mathrm{ddH}_{2} \mathrm{O}$. The peptides were eluted by $70 \%$ acetonitrile with $0.1 \%$ formic acid.

\subsection{LC-MS/MS Analyses}

Peptides were separated via an Acclaim $\mu$-Precolumn $(0.5 \times 3 \mathrm{~mm}$, particle size of $5 \mu \mathrm{m}$, and an inner diameter of $75 \mu \mathrm{m}$; Thermo Scientific, Waltham, MA, USA). The peptide solution ( $1 \mu \mathrm{g}$ of peptide in $1-4 \mu \mathrm{L}$ of $0.1 \%$ formic acid) was loaded on the column directly. The peptide concentration was performed in the isocratic mode of Mobile Phase C ( $2 \%$ acetonitrile, $0.1 \%$ formic acid) at a flow rate of $10 \mu \mathrm{L} / \mathrm{min}$ for $4 \mathrm{~min}$.

High-performance liquid chromatography (HPLC) was applied for peptide separation. The procedure was performed via Ultimate 3000 Nano LC System (Thermo Scientific, Waltham, MA, USA). C18 columns (Acclaim ${ }^{\circledR}$ PepMapTM RSLC with an inner diameter of $75 \mu \mathrm{m}$; Thermo Fisher Scientific, Waltham, MA, USA) were used for peptide separation. The peptides were eluted in a gradient mode. The gradient was formed by $0.1 \%$ formic acid 
(mobile phase A) and buffer B ( $80 \%$ acetonitrile, $0.1 \%$ formic acid) at flow rate of $0.3 \mu \mathrm{L} / \mathrm{min}$. The columns were equilibrated by mobile phase A for $12 \mathrm{~min}$ with a subsequent increase in the buffer B concentration from 5\% to 35\% for over $95 \mathrm{~min}$. Then, the buffer B concentration was increased to $99 \%$ (6 min). The columns were repeatedly washed with $99 \%$ buffer B and equilibrated with buffer A (7 min).

The MS analysis was performed in at least three technical replicates using a Q Exactive HF-X mass spectrometer (Q Exactive HF-X Hybrid Quadrupole-OrbitrapTM Mass Spectrometer, Thermo Fisher Scientific, Waltham, MA, USA). The analysis was performed under the following conditions: the capillary temperature was $240{ }^{\circ} \mathrm{C}$ and the ionizing voltage was $2.1 \mathrm{kV}$. The spectra were acquired at the $300-1500 \mathrm{~m} / \mathrm{z}$ range at a resolution of 120,000 (MS). Tandem spectra were acquired at the 140-2000 m/ $z$ range at a resolution of 15,000 (MS/MS). The maximum integration for precursor and fragment ions was 50 and $110 \mathrm{~ms}$, respectively. The AGC target for precursor and fragment ions were set to $1 \times 10^{6}$ and $2 \times 10^{5}$, respectively. An isolation intensity threshold of 50,000 counts was determined for the precursor's selection. The top 20 precursors were chosen for fragmentation with high-energy collisional dissociation (HCD) at 29 NCE. Precursors with a charge state of +1 and more than +5 were rejected, and all measured precursors were dynamically excluded from triggering a subsequent MS/MS for $20 \mathrm{~s}$.

Mass spectrometric measurements were performed using the equipment at the "Human Proteome" Core Facility of the Institute of Biomedical Chemistry (Russia, Moscow).

\subsection{Protein Identification}

Label-free quantitation using MaxQuant software (version 1.6.3.4) was applied to experimental data. Only proteins identified by two or more unique/razor peptides were considered for label-free quantitation. The identification was performed against a concatenated target/decoy version of the Homo sapiens complement of the UniProtKB (July 2021). The database search was performed under the following parameters: enzyme specificitytrypsin; maximum cleavages allowed-2; MS1 and MS2 tolerances-5.0 and 20 ppm, respectively; fixed modification—carbamidomethylation (Cys); variable modifications- $\mathrm{N}$ terminal proteins acetylation and methionine oxidation (Met), false discovery rate estimated using the decoy hit distribution for peptide spectrum matches (PSMs); peptides and proteins identification-1.0\%.

Author Contributions: Conceptualization: N.G.L. and A.L.R.; methodology: A.L.R., V.G.Z., M.N.K., D.S.L., D.D.R. and P.M.K.; investigation: V.G.Z., A.L.R., P.M.K., D.D.R., M.N.K., N.G.L. and O.V.T.; resources: A.L.R. and D.D.R.; data curation: V.G.Z. and D.D.R.; validation: A.L.R.; writing-original draft preparation: N.G.L., A.L.R., P.M.K., V.G.Z., D.D.R., M.N.K., D.S.L. and O.V.T.; writing一review and editing: N.G.L., A.L.R., P.M.K., D.D.R., M.N.K., D.S.L. and O.V.T.; supervision, N.G.L.; project administration: A.L.R. and N.G.L. All authors have read and agreed to the published version of the manuscript.

Funding: The work was conducted in the framework of the Russian Federation fundamental research program for the long-term period of 2021-2030.

Institutional Review Board Statement: Not applicable.

Informed Consent Statement: Not applicable.

Data Availability Statement: Proteomic data were deposited to the ProteomeXchange Consortium via the PRIDE partner repository (http:/ / www.proteomexchange.org/Project, accessed on 29 December 2021, accession: PXD030700).

Acknowledgments: Mass spectrometric measurements were performed using the equipment at the "Human Proteome" Core Facility of the Institute of Biomedical Chemistry (Russia, Moscow).

Conflicts of Interest: The authors declare no conflict of interest. The funders had no role in the design of the study; in the collection, analyses, or interpretation of data; in the writing of the manuscript or in the decision to publish the results. 


\section{References}

1. Truong, A.B.; Kretz, M.; Ridky, T.W.; Kimmel, R.; Khavari, P.A. P63 Regulates Proliferation and Differentiation of Developmentally Mature Keratinocytes. Genes Dev. 2006, 20, 3185-3197. [CrossRef] [PubMed]

2. Botchkarev, V.A.; Flores, E.R. P53/P63/P73 in the Epidermis in Health and Disease. Cold Spring Harb. Perspect. Med. 2014, 4, a015248. [CrossRef] [PubMed]

3. Westfall, M.D.; Mays, D.J.; Sniezek, J.C.; Pietenpol, J.A. The Delta Np63 Alpha Phosphoprotein Binds the P21 and 14-3-3 Sigma Promoters in Vivo and Has Transcriptional Repressor Activity That Is Reduced by Hay-Wells Syndrome-Derived Mutations. Mol. Cell. Biol. 2003, 23, 2264-2276. [CrossRef] [PubMed]

4. Baran, W.; Szepietowski, J.C.; Szybejko-Machaj, G. Expression of P53 Protein in Psoriasis. Acta Dermatovenerol. Alp. Pannonica Adriat. 2005, 14, 79-83.

5. Batinac, T.; Zamolo, G.; Jonjić, N.; Gruber, F.; Petrovecki, M. P53 Protein Expression and Cell Proliferation in Non-Neoplastic and Neoplastic Proliferative Skin Diseases. Tumori 2004, 90, 120-127. [CrossRef] [PubMed]

6. Pellegrini, G.; Dellambra, E.; Golisano, O.; Martinelli, E.; Fantozzi, I.; Bondanza, S.; Ponzin, D.; McKeon, F.; De Luca, M. P63 Identifies Keratinocyte Stem Cells. Proc. Natl. Acad. Sci. USA 2001, 98, 3156-3161. [CrossRef] [PubMed]

7. Lehman, T.A.; Modali, R.; Boukamp, P.; Stanek, J.; Bennett, W.P.; Welsh, J.A.; Metcalf, R.A.; Stampfer, M.R.; Fusenig, N.; Rogan, E.M. P53 Mutations in Human Immortalized Epithelial Cell Lines. Carcinogenesis 1993, 14, 833-839. [CrossRef] [PubMed]

8. Xu, J.; Reumers, J.; Couceiro, J.R.; De Smet, F.; Gallardo, R.; Rudyak, S.; Cornelis, A.; Rozenski, J.; Zwolinska, A.; Marine, J.-C.; et al Gain of Function of Mutant P53 by Coaggregation with Multiple Tumor Suppressors. Nat. Chem. Biol. 2011, 7, 285-295. [CrossRef] [PubMed]

9. Cordani, N.; Pozzi, S.; Martynova, E.; Fanoni, D.; Borrelli, S.; Alotto, D.; Castagnoli, C.; Berti, E.; Viganò, M.A.; Mantovani, R. Mutant P53 Subverts P63 Control over KLF4 Expression in Keratinocytes. Oncogene 2011, 30, 922-932. [CrossRef] [PubMed]

10. Neilsen, P.M.; Noll, J.E.; Suetani, R.J.; Schulz, R.B.; Al-Ejeh, F.; Evdokiou, A.; Lane, D.P.; Callen, D.F. Mutant P53 Uses P63 as a Molecular Chaperone to Alter Gene Expression and Induce a Pro-Invasive Secretome. Oncotarget 2011, 2, 1203-1217. [CrossRef] [PubMed]

11. Martynova, E.; Pozzi, S.; Basile, V.; Dolfini, D.; Zambelli, F.; Imbriano, C.; Pavesi, G.; Mantovani, R. Gain-of-Function P53 Mutants Have Widespread Genomic Locations Partially Overlapping with P63. Oncotarget 2012, 3, 132-143. [CrossRef] [PubMed]

12. Jung, Y.-S.; Qian, Y.; Yan, W.; Chen, X. Pirh2 E3 Ubiquitin Ligase Modulates Keratinocyte Differentiation through P63. J. Investig. Dermatol. 2013, 133, 1178-1187. [CrossRef] [PubMed]

13. Fuchs, E. Scratching the Surface of Skin Development. Nature 2007, 445, 834-842. [CrossRef] [PubMed]

14. Brinkman, E.K.; Chen, T.; Amendola, M.; van Steensel, B. Easy Quantitative Assessment of Genome Editing by Sequence Trace Decomposition. Nucleic Acids Res. 2014, 42, e168. [CrossRef] [PubMed]

15. Wessel, D.; Flügge, U.I. A Method for the Quantitative Recovery of Protein in Dilute Solution in the Presence of Detergents and Lipids. Anal. Biochem. 1984, 138, 141-143. [CrossRef]

16. Rappsilber, J.; Mann, M.; Ishihama, Y. Protocol for Micro-Purification, Enrichment, Pre-Fractionation and Storage of Peptides for Proteomics Using StageTips. Nat. Protoc. 2007, 2, 1896-1906. [CrossRef] [PubMed] 